Audiology

Neurotology
Audiol Neurotol 2018;23:126-134

DOI: $10.1159 / 000491991$
Received: February 6, 2018

Accepted after revision: July 6, 2018

Published online: September 18, 2018

\title{
Pediatric Auditory Brainstem Implantation: Impact on Audiological Rehabilitation and Tonal Language Development
}

\author{
John Ka Keung Sung Betty Pui Ki Luk Terence Ka Cheong Wong \\ Jiun Fong Thong Hoi Tung Wong Michael Chi Fai Tong \\ Department of Otorhinolaryngology, Head and Neck Surgery, The Chinese University of Hong Kong, \\ Hong Kong, China
}

\section{Keywords \\ Auditory brainstem implant . Cochlear implant - Pediatric . Children · Deafness · Hearing loss - Auditory rehabilitation . Speech development $\cdot$ Tonal language and speech perception category}

\begin{abstract}
Objective: This is a retrospective review of the impact of an Auditory Brainstem Implant (ABI) on the audiological rehabilitation and tonal language development of pediatric patients with prelingual profound deafness in Hong Kong. Results: From January 2009 to February 2015, 11 pediatric patients with profound prelingual deafness received an $A B I$ in Hong Kong (age range 1.67-3.75 years). Etiologies included Cochlear Nerve Deficiency in 7, Severe Cochlear Malformations in 2, and Retrocochlear Deafness in 2. All of them were rehabilitated in Cantonese, a dialect of Chinese. Standard pediatric cochlear implant outcome measurements were used in this study that comprised of the 7-Sound Detection, Syllable Identification, Vowel Identification, Consonant Identification, Tone Imitation, Tone Production and Speech Perception Category. Audiological rehabilitation and speech development outcomes were reviewed. Age-matched outcomes of pediatric cochlear implant users were used for
\end{abstract}

comparisons. Conclusion: Encouraging results of speech development were found, especially with continued use of the $\mathrm{ABI}$. There was considerable variation in outcomes. Children with coexisting developmental and nonauditory cognitive disabilities did not perform as well. Auditory brainstem implantation is a safe and beneficial treatment for profound prelingual deafness in Cantonese-speaking pediatric patients.

(C) 2018 The Author(s)

Published by S. Karger AG, Basel

\section{Introduction}

Cochlear implants (CI) have revolutionized the treatment of prelingual profound deafness. However, where cochlear implantation is contraindicated or has failed remains a challenging clinical dilemma for hearing health professionals worldwide. Auditory brainstem implants (ABI) were first developed for the management of neurofibromatosis type 2 in adults by House and Hitselberger

This review article is conducted and edited according to the principles expressed in the Declaration of Helsinki. This Research is NOT funded by any third parties and there is NO conflict of interests to declare.

\begin{tabular}{ll}
\hline KARGER & $\begin{array}{l}\text { (c) } 2018 \text { The Author(s) } \\
\text { Published by S. Karger AG, Basel }\end{array}$ \\
E-Mail karger@karger.com & $\begin{array}{l}\text { This article is licensed under the Creative Commons Attribution- } \\
\text { NonCommercial-NoDerivatives 4.0 International License (CC BY- } \\
\text { www.karger.com/aud }\end{array}$ \\
$\begin{array}{l}\text { NC-ND) (http://www.karger.com/Services/OpenAccessLicense). } \\
\text { Usage and distribution for commercial purposes as well as any dis- } \\
\text { tribution of modified material requires written permission. }\end{array}$
\end{tabular}


in 1979 [Edgerton and House, 1982; Hitselberger et al., 1984]. Subsequently, they were used in other non-tumor inner ear diseases and deformities such as cochlear aplasia, labyrinthine aplasia [Michel deformity], and cochlear nerve aplasia [Colletti et al., 2009]. With the pioneering work of Colletti and others, the indication for auditory brainstem implantation was extended to the treatment of pediatric prelingual deafness [Colletti et al., 2002; Colletti et al., 2005; Colletti, 2008; Sennaroglu et al., 2009; Choi et al., 2011].

We present the audiological and tonal language developmental outcomes of pediatric patients with ABIs followed-up for 1-5 years, using age-matched outcomes of CI users for comparison. To the best of our knowledge, this is the first manuscript describing the outcomes of pediatric $\mathrm{ABI}$ users in a tonal language (Cantonese) setting.

\section{Material and Methods}

\section{Patient Demographics}

Between January 2009 and February 2015, 11 prelingually deaf children who either failed or had contraindications for cochlear implantation received an $\mathrm{ABI}$ in our unit. The age at implantation ranged from 1.67 to 3.75 years (mean 2.67 years). There were 8 males and 3 females. Etiologies included cochlear nerve deficiency $(n=7)$ and severe cochlear malformations $(n=2)$ as shown radiologically. In the cochlear nerve deficiency group, 2 of the 7 had coexisting Auditory Neuropathy Spectrum Disorder (ANSD) features on their Auditory Brainstem Response (ABR) study. Retrocochlear deafness $(n=2)$ was defined as a normal cochlear nerve radiologically and the presence of only waves I and II in ABR (Table 1). Retrocochlear deafness and ANSD are elaborated in the discussion section below.

\section{Preoperative Assessment}

All subjects were initially identified by the Hong Kong-wide universal newborn hearing screening program and were referred to our unit for further assessment. A battery of tests, including $\mathrm{ABR}$, was performed to assess their auditory threshold and pathway. The need for cochlear implantation was decided after a hearing aid trial of at least 3 months. Both CT scan and MRI of the inner ear were performed to assess their anatomy. Children who met the criteria for cochlear implantation would proceed to CI surgery. Children who showed limited or no benefit from hearing aids, and who had abnormalities on imaging such as cochlear aplasia or severe malformations would be further assessed for ABI candidacy. We did not use evoked Auditory Brainstem Response (eABR) as a standard preoperative assessment in this group of pediatric patients, as the sensitivity and specificity of the test are unknown. It was technically challenging to differentiate a positive eABR response from an artifact. The decision to proceed with cochlear implantation was jointly made with the parents in view of the lack of benefit from aiding and speech and language development. Although we performed an eABR under general anesthesia for subject 8 , no positive responses could be

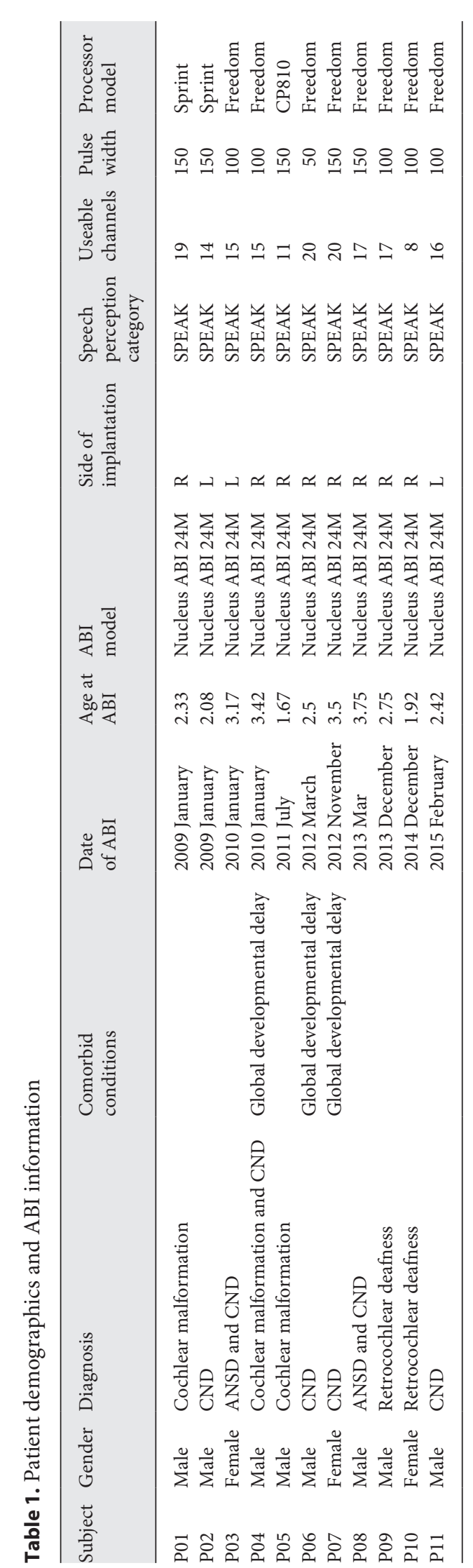

Impact on Audiological Rehabilitation and Tonal Language Development 
obtained. In spite of this, we proceeded to a CI as the first-line measure for the subject. Surgery was uneventful, but he derived no benefit from the CI.

\section{ABI Surgery}

A minimally invasive retrosigmoid approach with a $3-4 \mathrm{~cm}$ vertical post-auricular incision and a mini-craniotomy of $2-3 \mathrm{~cm}$ in diameter for electrode placement was employed with intraoperative monitoring of cranial nerves VII-XI in all cases [Colletti, 2010]. Optimal positioning of the electrode was confirmed by intraoperative Electrically eABR.

\section{Audiological Perception Outcomes}

Each subject's auditory perception ability was tested using the Cantonese Basic Speech Perception Test (CBSPT) before cochlear implantation, and regularly afterwards too. Three domains of auditory perception were tested:

1. Sound detection (7-sound detection) - the ability to detect the Ling's seven sounds in a quiet environment.

2. Suprasegmental (syllable identification) - the ability to identify the number of syllables in a sound string.

3. Segmental.

- Vowel identification - the ability to identify a word with an appropriate vowel in an array which is different from the vowel only.

- Consonant identification - the ability to identify a word with an appropriate consonant in an array which is different from the consonant only.

Raw scores of these domains were used to determine the subject's speech perception category (SPC) from 0 to 7 (Table 2). The CBSPT only covers the test scope up to consonant perception. For patients scoring over $75 \%$ in the consonant identification, open set word recognition and sentence recognition tests were used to assess higher levels of speech perception ability.

Tone imitation and production tests were used to provide additional information on this specific group of Cantonese speaking children. Tone imitation tests the ability to imitate words with different Cantonese tones while tone production assesses the ability to produce words with different Cantonese tones.

The age-matched group consists of 17 children implanted between the ages of 1.09 and 3.08 years. All of these demonstrated no significant developmental delay. They were identified with severe to profound hearing loss with no indication of ANSD. Imaging, including CT and MRI, suggested no significant abnormalities in the cochlear and internal auditory meatus. They demonstrated no significant developmental delay except in the areas of speech and language.

\section{Results}

\section{Surgical Outcomes and Complications}

No major complications occurred in this series of pediatric patients. One subject in the series (Subject 10) developed deterioration of performance and nonauditory stimulation (coughing) with use of the ABI device about 12 months postoperatively. Revision ABI surgery was
Table 2. Speech perception categories (Lee et al., 2006)

\begin{tabular}{ll}
\hline $\begin{array}{l}\text { Speech } \\
\text { perception } \\
\text { category }\end{array}$ & Definition \\
\hline 0 & Minimal sound detection \\
1 & Sound detection \\
2 & Suprasegmental perception \\
3 & Vowel perception \\
4 & Consonant perception \\
5 & 10-20\% open-set word recognition \\
6 & 20-50\% open-set word recognition \\
7 & $>50 \%$ open-set word recognition \\
\hline
\end{tabular}

performed and lateral and inferior displacement of the electrode was found intraoperatively, which was successfully corrected. There was some initial improvement after the revision surgery, but ultimately no consistent sound detection could be identified.

We had performed cochlear implantation on 7 of the 11 subjects. Subject 1 had a hypoplastic middle ear with an unsuccessful electrode placement attempt. The remaining 6 subjects, $2,3,6,7,8$, and 11 , who underwent uneventful surgery with satisfactory electrode position, showed neither consistent sound detection nor benefit in speech and language development after 1 year of CI use with regular auditory programming attempts and speech training. No cochlear implantation was considered for the other 4 of the 11 subjects, as they all had severe cochlear malformations (subject 4 and 5) or retrocochlear deafness (subjects 9 and 10). For the 2 subjects with retrocochlear deafness, the decision of not to implant them with a CI was made in conjunction with parental preference.

In a majority of subjects, 7-Sound detection could be achieved at a relatively early stage postoperatively and this is comparable to age-matched CI users. Five subjects scored $100 \%$ with a mean score of $92.6 \%$ (range 55.6$100 \%$ ) at 1 year postoperatively (Fig. 1). For Syllable Identification, 4 achieved $100 \%$ with a mean score of $77.8 \%$ (range $0-100 \%$ ), and this happened more gradually over the first 2-3 years (Fig. 2). For Vowel Identification, the average score was $59.1 \%$ (range $16.7-87.5 \%$; Fig. 3). For Consonant Identification, the average score was $62.7 \%$ (range 0-87.5\%; Fig. 4). As shown in Figures 2-4, Syllable Identification, Vowel Identification, and Consonant Identification were achieved by a majority of our subjects albeit at a relatively slower rate when compared to CI users. However, whereas outcomes of CI users plateaued after 
Fig. 1. 7-Sound detection.

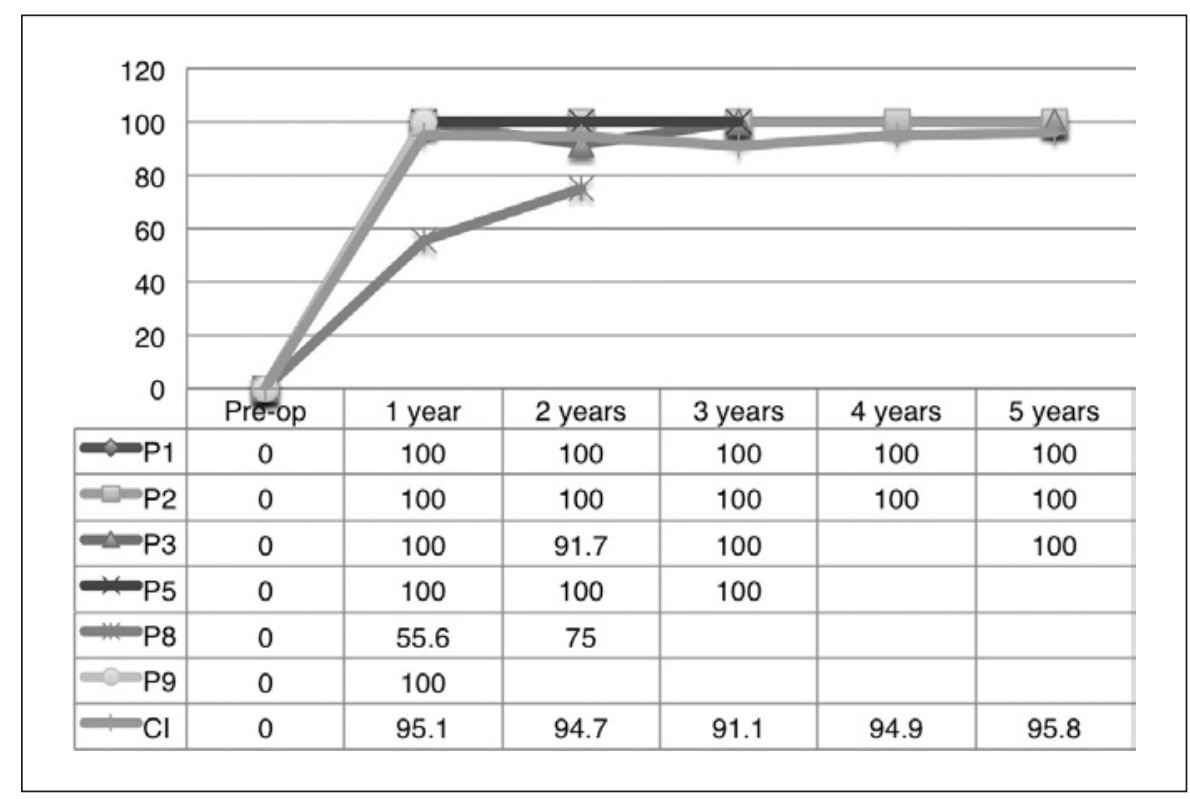

Fig. 2. Syllable identification.

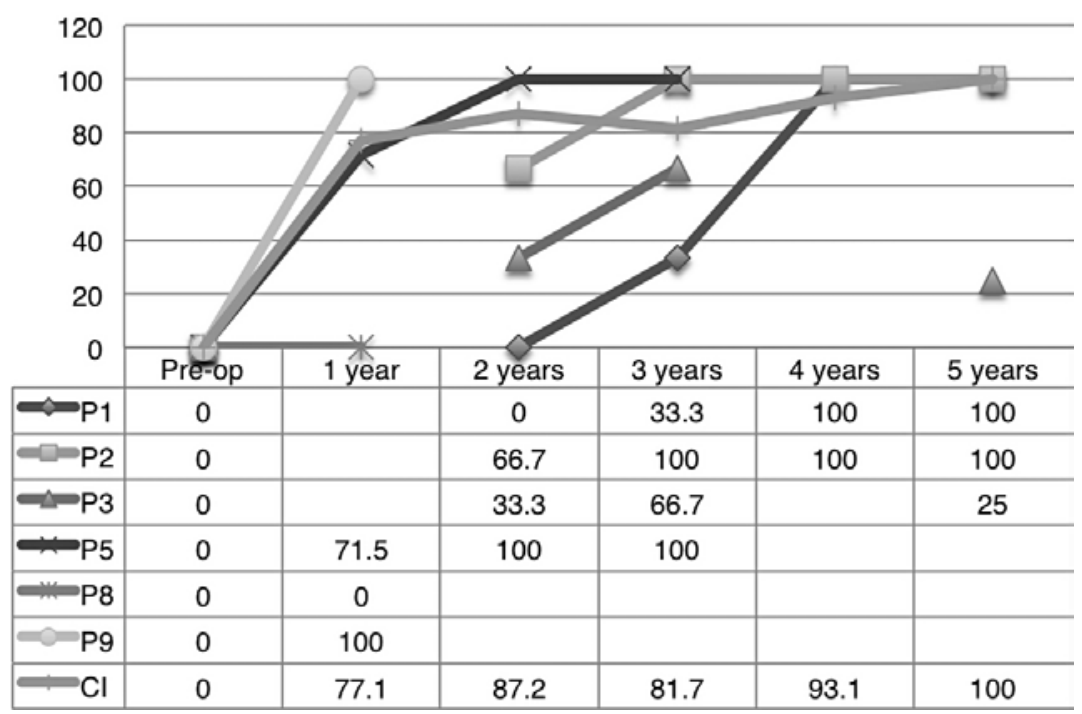

2-3 years, ABI subjects appeared to continue to show improvement even 5 years after surgery. For Tone Imitation, the average score was $52.9 \%$ (range $0-88.9 \%$; Fig. 5). For Tone Production, the average score was $63.6 \%$ (range 0-96\%; Fig. 6). The Tone Imitation and Production scores are even closer to CI users, with good results achieved by some of the subjects who had less impressive outcomes in other parameters such as the SPC [Lee et al., 2006].

At the time of data collection, 2 subjects achieved Category 5-7 in the SPC [Lee et al., 2006] assessment, which is equivalent to open-set word recognition (Table 2). Two subjects achieved Category 4 and the remaining 2 subjects Category 1-3 (Fig. 7). Similar to the other outcome measurement parameters, speech perception represented by the SPC showed a slow but steadily improving trend during the follow-up period. Four of the 11 subjects achieved SPC 4 or better. With the current trend, we expect some of the subjects to achieve higher categories at the end of the 5 year postoperative follow-up period. 
Fig. 3. Vowel identification.

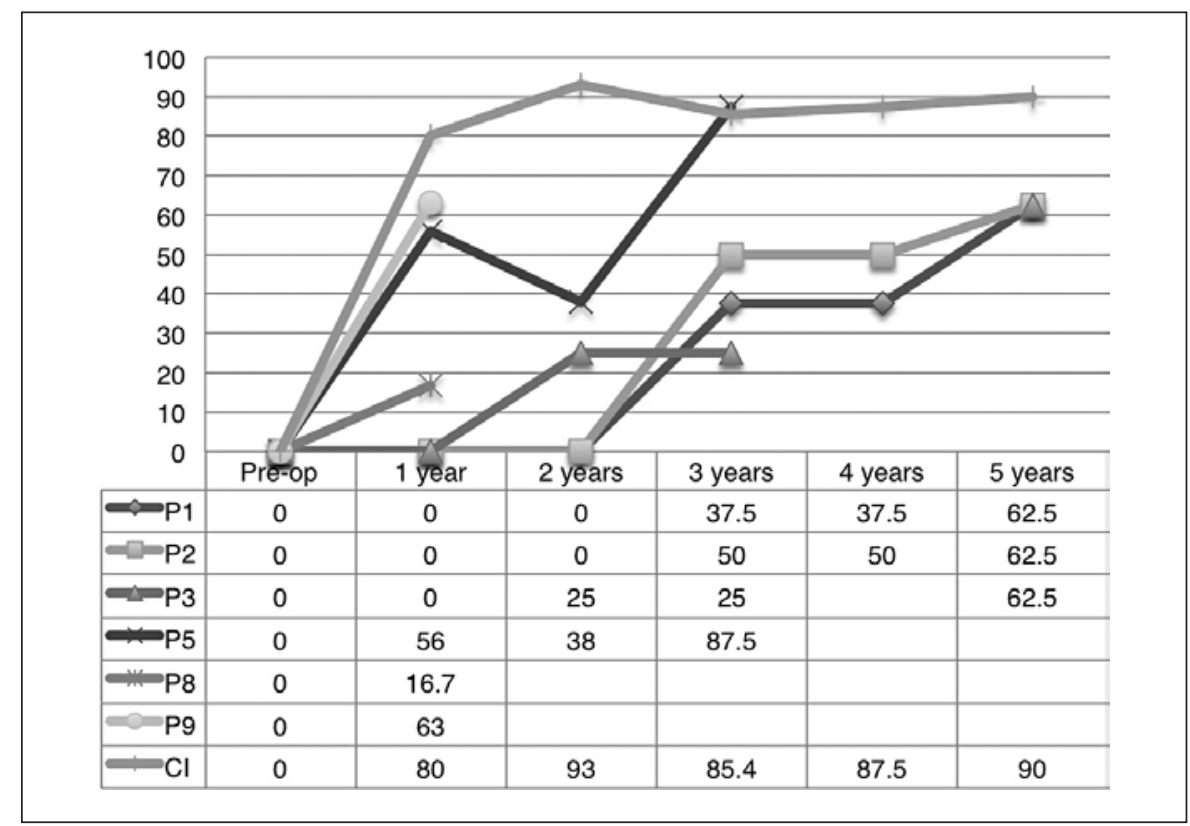

Fig. 4. Consonant identification.

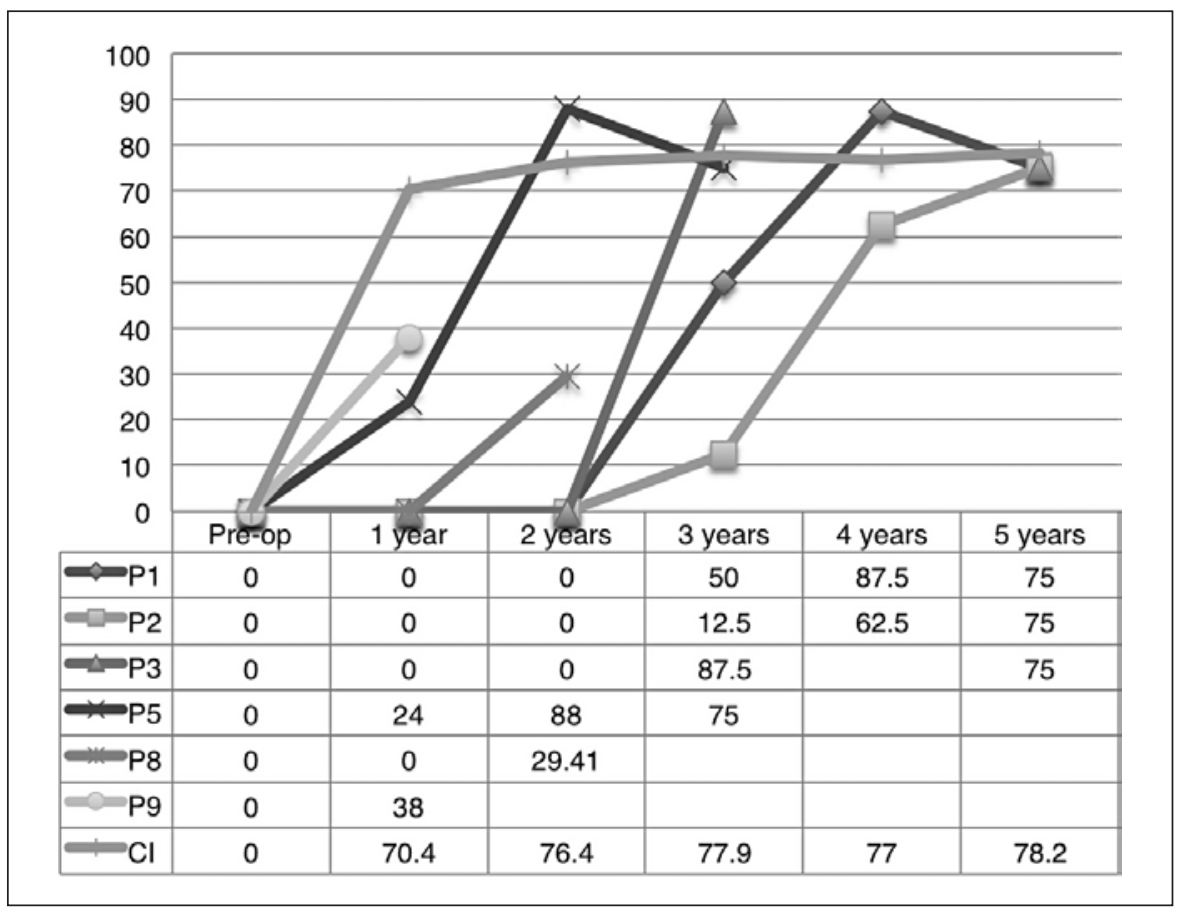

Subjects 4, 6, and 7 did not perform well enough to complete our outcome assessment. Of note, these subjects have coexisting nonauditory developmental disabilities. Nevertheless, parents reported good compliance to ABI usage by their children who demonstrated improved awareness to environmental sounds.

\section{Discussion}

Prolonged Time Frame of Auditory Development

Good sound detection could be achieved in the first year for ABI users and this is comparable to age-matched CI users and published case series data [Colletti et al., 2014a]. CI users can typically achieve good outcomes in 
Fig. 5. Tone imitation.

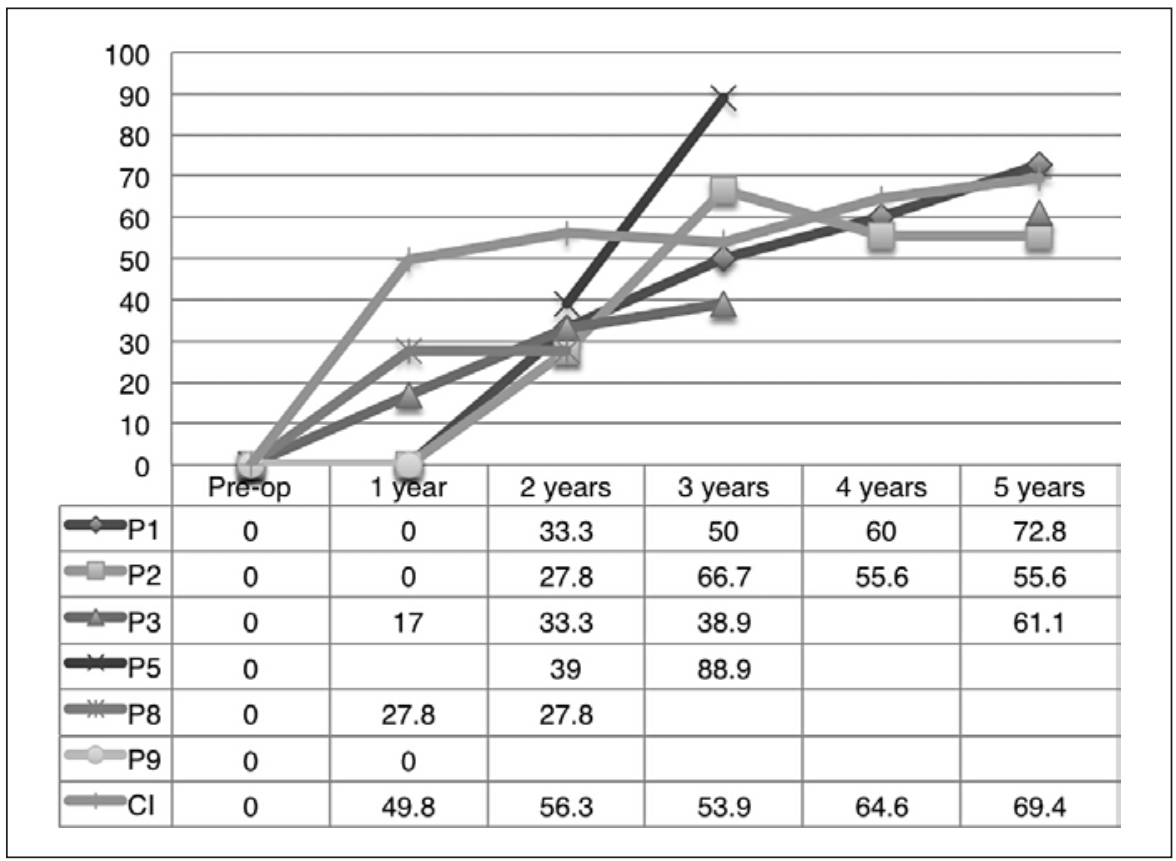

Fig. 6. Tone production.

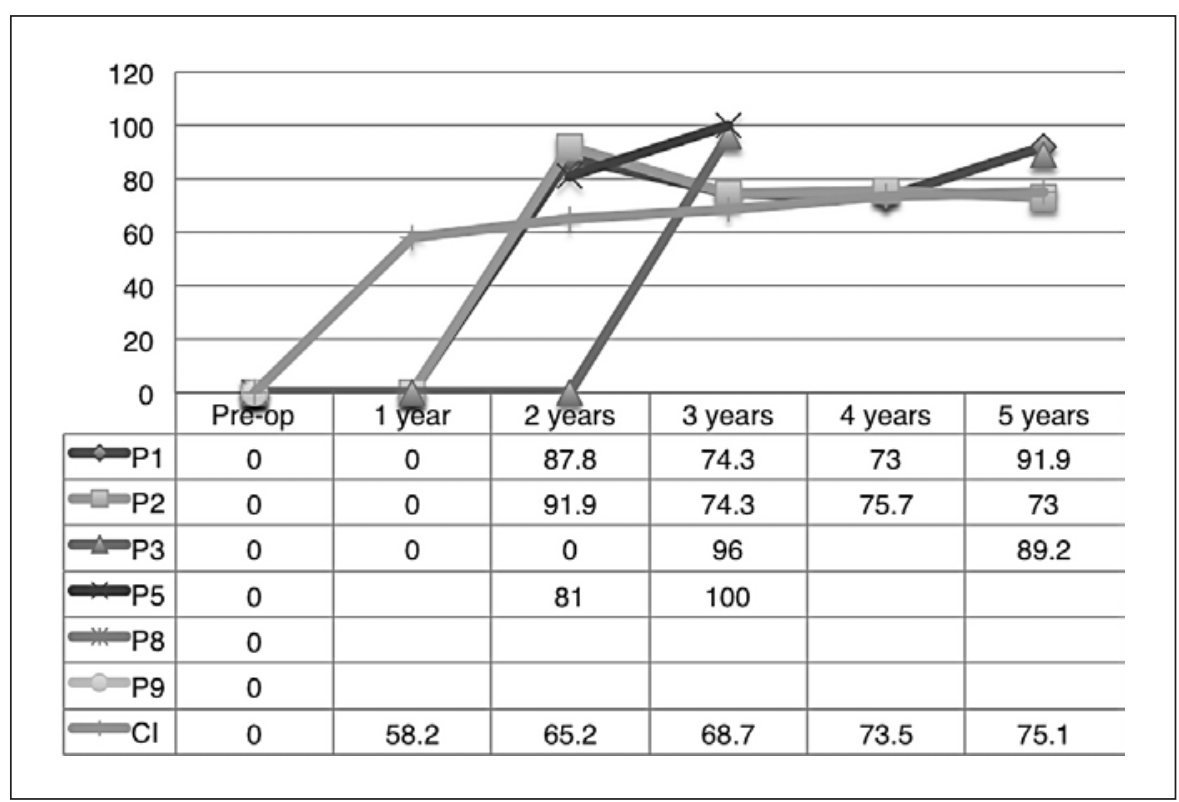

the SPC, and Tone Imitation and Production within 2 years of surgery [Niparko et al., 2010]. Although our current data show a slower progress compared to CI users, there is continued improvement in all parameters during the 5 year follow-up period. This is consistent with the findings of other authors, who also found that additional comorbid handicaps slow down progression [Yucel et al., 2015].

Impact on Audiological Rehabilitation and Tonal Language Development
Poor (Slower) Speech Perception and

Tonal Production Outcomes in Subjects with Relatively Well-Developed Environmental Sound Detection

Most of the subjects were able to detect sounds and did well in environmental sound detection tasks. However, their overall performance in speech perception was poorer than that of the CI children. The different location of 
Fig. 7. Speech perception category.

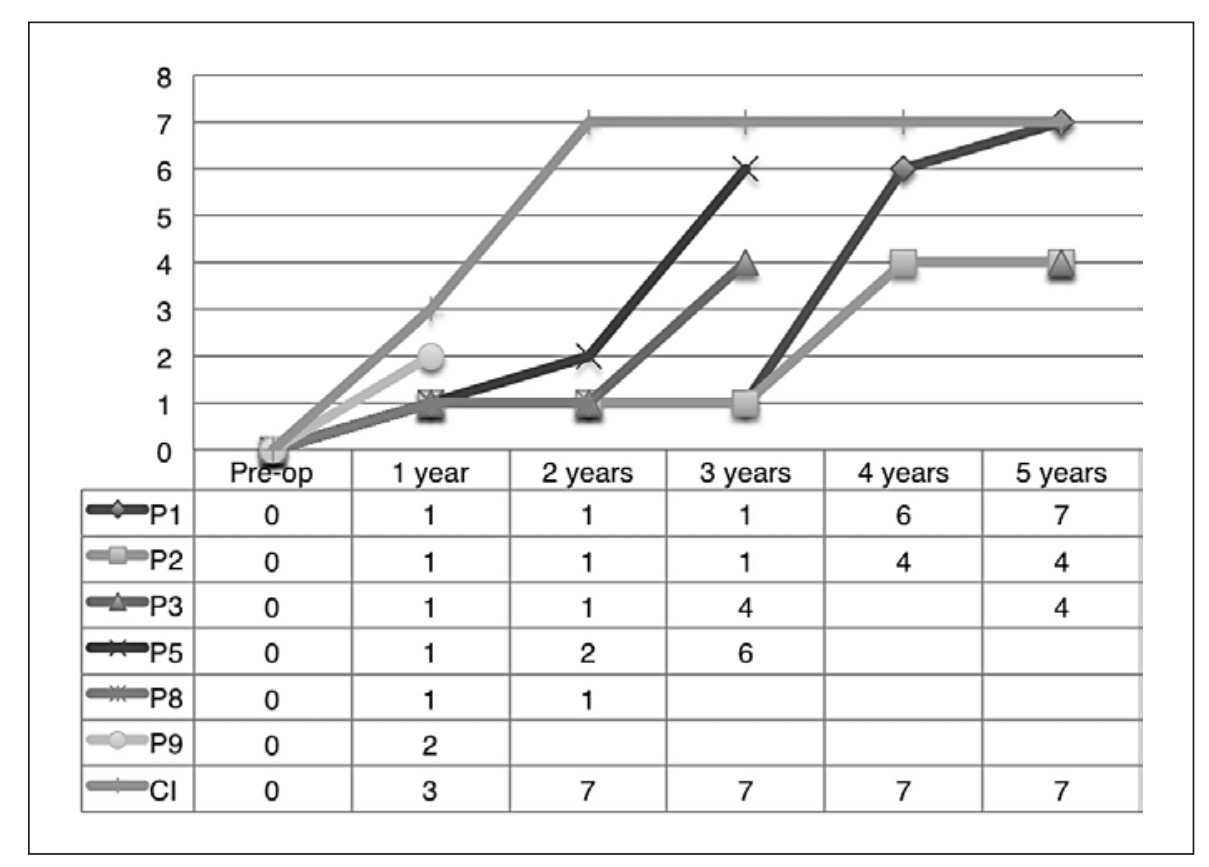

electrodes between $\mathrm{CI}$ and ABI, and the lack of intracochlear tonotopic arrangement of the ABI electrode might have contributed to the difference in the ability to perceive the temporal and spectral information necessary for speech perception [Colletti and Shannon, 2005; Bayazit et al., 2014].

\section{Comorbid Nonauditory and Cognitive Conditions}

In our series, there was significant variability in outcomes with open-set speech perception and minimal sound detection between the subjects. Pediatric performance might be strongly dependent on etiology of deafness, cognitive ability, and the integrity of the auditory pathways. Subjects with an auditory nerve and a cochlear malformation performed better than subjects with either a cochlear nerve deficiency or an ANSD. Similar to the pediatric CI population, subjects with multiple disabilities showed slower progress and limited benefit from an ABI. The poor open-set speech recognition and speech production associated with cognitive comorbidity suggest that disorders of central auditory signaling or speech and language processing constitute an important factor in ABI outcome [Colletti et al., 2014a; Pisoni, 2000]. Despite relatively poor audiological outcomes, the families of this group of children reported good compliance and noticeable benefits of device use in training and daily activities.

\section{Neural Plasticity}

Despite the high variability of ABI outcomes and the associated time-frame, an early decision to convert from a CI to an ABI is advocated. We agree with other researchers who advocate that ABI should be considered for optimal auditory perception and speech and language development when a lack of progress with a CI is evident [Colletti et al., 2014a].

\section{Etiologies and Outcomes}

The etiology of deafness is the main determining factor of outcome. In our series, the 2 outstanding performers (subjects 1 and 5) had major middle ear and inner ear deformities. The structural and functional integrity of the cochlear nerve and cochlear nucleus are important prognostic factors for successful auditory rehabilitation, especially for open-set word recognition. The other subjects in our series with ANSD with Cochlear Nerve Deficiency and Retrocochlear Deafness had less satisfactory outcomes.

From a review of outcomes from different international centers [Sennaroglu et al., 2009; Couloigner et al., 2014] and ours, we note that results obtained are substantially better in the presence of a functional cochlear nerve (e.g., hearing loss due to inner ear malformations or meningitis), and so we hypothesize that the cochlear nuclei in these children might be present and functional. However, in those children with an abnormal hearing 
nerve (so-called neural deafness or retrocochlear deafness), the nerve might be dysfunctional, thereby preventing good signal transmission to the central auditory pathways, resulting in a poor clinical outcome with ABI. It would seem that in order to achieve efficient word recognition, ABI requires a functionally intact neural pathway. If this is absent, either anatomically or functionally, the stimulation in the auditory cortex may not be sufficient to result in significant open-set speech discrimination.

ANSD has wide spectrum of clinical presentations. In our unit, 38 cases were identified from January 2009 to February 2015, either in one ear or in both ears, with the presence of cochlear microphonics but with an absence of ABR. Ten out of 38 derived no benefit from bilateral amplification and so we proceeded with cochlear implantation. Eight of them were able to develop differing levels of speech and language after cochlear implantation. However, 2 of them (subjects 3 and 8), in addition to ANSD, also had cochlear nerve deficiencies that might have favored the poorer outcomes, and these 2 subjects did not show consistent sound detection at home, at school, or at the clinic after cochlear implantation. Different programming parameters were attempted and auditory training was provided. After at least 1 year of cochlear implant use, these subjects proceeded to auditory brainstem implantation, as there was no observed improvement in their speech and language development. An ANSD with a coexisting MRI finding of an abnormal cochlear nerve or of a cochlear nerve deficiency is a poor prognostic factor for cochlear implant outcome.

A cochlear nerve deficiency is a radiological diagnosis made on MRI or CT scans. Other inner ear malformations often coexist (e.g., cochlear malformations and a large vestibular aqueduct syndrome) and must be taken into consideration. In our series, we had 7 subjects with abnormal cochlear nerve anatomy on MR imagining. Within this group, subjects 3 and 8 had coexisting ABR findings of an ANSD. CND is not an absolute contraindication for cochlear implantation unless there is a coexisting inner or middle malformation. A recently published consensus statement on decision making for CI and ABI [Sennaroglu, 2016], stated that ideally an electrically eABR should be performed before CI or ABI when there is a suspicion of a hypoplastic cochlear nerve. A positive $\mathrm{eABR}$ response would sway the implant team to choose $\mathrm{CI}$ rather than $\mathrm{ABI}$, while the absence of responses would need to be correlated with imaging findings.

According to the Guidelines for the Assessment and Management of ANSDs in Young Infants (NHSP ANSD
Guidelines version 2.2, 2013) [Norris et al., 2014], ANSD is the name used to describe the condition in which an individual has otoacoustic emissions and/or cochlear microphonics but does not have or has severely abnormal ABRs. In our series, Retrocochlear Deafness was diagnosed by ABR using waves I and II. We separated it from ANSD, as it suggests a lesion higher than the auditory nerve in the auditory pathway. Contrary to other subjects in our series with cochlear nerve deficiency and inner deformities, both of the patients with retrocochlear deafness have normal inner ear and cochlear nerve anatomy by MR imaging. There is no consensus on the classification of this category of patients and their clinical management is controversial. No conclusions could be drawn from the present study regarding the surgical management of this group of patients.

\section{Use of Adjuncts - Sign Language and Lip-reading}

The group of children that we have been describing is usually implanted with an ABI at an older age than children who receive cochlear implants. With a later starting point and slower progress, as we have reported in this study, auditory stimulation alone appears to be insufficient for development. We strongly recommend additional modes of communication to facilitate the overall development of children who find themselves in this group. Extensive counseling of parents emphasizing the importance of additional modalities of communication is essential. Bilingual programs and lip-reading are 2 major adjuncts advocated for the children in our study. The 3 subjects in our study with coexisting developmental disability reported good compliance and benefit from $A B I$ usage in their rehabilitation. $A B I$ is an important adjunct to the child's cognitive and language development, where lip-reading and signing are incorporated.

\section{Conclusion}

Although this study is limited by a small sample size and a retrospective nature, our results show that with persistent device use, meaningful auditory perception can develop in most subjects. For children who fail to show benefit from a CI, an early switch to an $\mathrm{ABI}$ is important to maximize their speech development. While the cause of deafness is the main determining factor for outcome, the presence of additional nonauditory and cognitive disabilities will have a negative impact on it. 


\section{References}

Bayazit YA, Kosaner J, Cinar BC, et al: Methods and preliminary outcomes of pediatric auditory brainstem implantation. Ann Otol Rhinol Laryngol 2014;123:529-536.

Choi JY, Song MH, Jeon JH, et al: Early surgical results of auditory brainstem implantation in nontumor patients. Laryngoscope 2011;121: 2610-2618.

Colletti L, Colletti G, Mandala M, et al: The therapeutic dilemma of cochlear nerve deficiency: cochlear or brainstem implantation? Otolaryngol Head Neck Surg 2014a;151:308-314.

Colletti L, Shannon RV, Colletti V, et al: The development of auditory perception in children after auditory brainstem implantation. Audiol Neurotol 2014b;19:384-394.

Colletti L, Zoccante L: Nonverbal cognitive abilities and auditory performance of children fitted with auditory brainstem implants: preliminary report. Laryngoscope 2018;118:14431448.

Colletti V, Carner M, Fiorino F, et al: Hearing restoration with auditory brainstem implant in three children with cochlear nerve aplasia. Otol Neurotol 2002;23:682-613.

Colletti V, Carner M, Miorelli V, et al: Auditory brainstem implant (ABI): new frontiers in adults and children. Otolaryngol Head Neck Surg 2005;133:126-138.

Colletti V, Shannon RV: Open set speech perception with auditory brainstem implant? Laryngoscope 2005;115:1974-1578.
Colletti V, Shannon R, Carner M, et al: Outcomes of non-tumor adults fitted with the auditory brainstem implant: 10-years' experience. Otol Neurotol 2009;30:614-618.

Colletti V, Shannon RV, Carner M, Veronese S, Colletti L: Complications in auditory brainstem implant surgery in adults and children. Otol Neurotol 2010;31:558-564.

Couloigner V, Gratacap M, Ambert-Dahan E, Borel S, Ettienne V, Kerouedan A, Bouccara D, Zerah M, Kalamarides M, Sterkers O: Implantation auditive du tronc cerebral chez l'enfant. A propos de trois cas (a report of three cases and review of auditory brainstem implants in children). Neurochirurgie 2014; 60:17-26.

Edgerton BJ, House WF, Hitselberger WE: Hearing by cochlear nucleus stimulation in humans. Ann Otol Rhinol Laryngol 1982;91: 117-124.

Hitselberger WE, House WF, Edgerton BJ, et al: Cochlear nucleus implants. Otolaryngol Head Neck Surg 1984;92:52-54.

Lee KYS, et al: Cantonese Basic Speech Perception Test. The Chinese University of Hong Kong, 2006.

Niparko JK, Tobey EA, Thal DJ, et al: Spoken language development in children following cochlear implantation. JAMA 2010;303:14981506.

Noiji KS, Kozin ED, Sethi R, et al: Systematic review of nontumor pediatric auditory brainstem implant outcomes. Otolaryngol Head Neck Surg 2015;153:739-750.
Norris LW, Velenovsky DS: Auditory neuropathy spectrum disorder: a review. J Speech Lang Hear Res 2014;57:1564-1576.

Pisoni DB: Cognitive factors and cochlear implants: some thoughts on perception, learning, and memory in speech perception. Ear Hear 2000;21:70-78.

Sennaroglu L, Ziyal I, Atas A, Sennaroglu G, Yucel E, Sevinc S, Ekin MC, Sarac S, Atay G, Ozgen B, Ozcan OE, Belgin E, Colletti V, Turan: Preliminary results of auditory brainstem implantation in prelingually deaf children with inner ear malformations including severe stenosis of the cochlear aperture and aplasia of the cochlear nerve. Otol Neurotol 2009;30: 708-715.

Sennaroglu L, Ziyal I, Atas A, et al: Preliminary results of auditory brainstem implantation in prelingually deaf children with inner ear malformations including severe stenosis of the cochlear aperture and aplasia of cochlear nerve. Otol Neurotol 2009;30:708-715.

Sennaroglu L, Colletti V, Lenarz T, et al: Consensus statement: Long-term results of $\mathrm{ABI}$ in children with complex inner ear malformations and decision making between CI and ABI. Cochlear Implants International 2016; 17:163-171.

Yucel E, Aslan F, Ozkan HB, et al: Recent rehabilitation experience with pediatric $\mathrm{ABI}$ users. J Int Adv Otol 2015;11:110-113. 